\title{
Homotopia Intervalar
}

E.S. PALMEIRA ${ }^{1}$, Departamento de Ciências Exatas e Tecnológicas, DCET, UESC - Universidade Estadual de Santa Cruz, 45662-900 Ilhéus, BA, Brasil.

B.R.C. BEDREGAL ${ }^{2}$, R.H.N. SANTIAGO ${ }^{3}$, Departamento de Informática e Matemática Aplicada, DIMAp, UFRN - Universidade Federal do Rio Grande do Norte, 59072-970 Natal, RN, Brasil.

Resumo. Nesse trabalho apresentamos uma formalização do conceito matemático de homotopia mergulhado no ambiente das funções intervalares definidas sobre o conjunto dos intervalos com extremos reais $\mathbb{I}(\mathbb{R})$, tanto considerando a Scottcontinuidade quanto a Moore-continuidade.

Definimos o que é uma homotopia Scott intervalar, bem como o que é uma homotopia Moore intervalar. Em seguida mostramos que esses dois conceitos coincidem para representações canônicas. Para finalizar, mostramos alguns resultados envolvendo homotopia intervalar e conceitos relacionados, tais como composição de homotopias e retrato por deformação.

Palavras-chave. Homotopia, Matemática Intervalar, Representação Intevalar.

\section{Introdução}

Uma das primeiras pessoas a usar intervalos para descrever resultados de medições de distância foi Norbert Wiener, em 1914. Além dele, Burkill [3], em 1924, e Young [18], em 1931, descreveram conceitos de aritmética intervalar. No entanto, os primeiros registos de trabalhos independentes devem-se a P. S. Dwyer [5], M. Warmus [17], T. Sunaga [14] e R. E. Moore [7]. Mas quem é considerado "o pai"da matemática intervalar é Moore, devido a sua grande contribuição para essa teoria através de diversos artigos e em particular de sua monografia [8], onde ele introduz a análise intervalar $[6,9,11]$.

Em sua essência, a matemática intervalar busca resolver problemas que se concentram fundamentalmente em dois aspectos: na criação de um modelo computacional que reflita de maneira fiel o controle e análise dos erros que ocorrem no processo computacional, bem como na escolha de técnicas de programação adequadas para desenvolvimento de softwares eficazes na minimização de erros nos resultados.

Com o intuito de desenvolver novas técnicas dentro da matemática intervalar é que propusemos, nesse trabalho, um estudo do conceito de homotopia (veja $[4,10$,

\footnotetext{
${ }^{1}$ espalmeira@uesc.br;

${ }^{2}$ bedregal@dimap.ufrn.br;

${ }^{3}$ regivan@dimap.ufrn.br;
} 
16]) mergulhada no ambiente da matemática intervalar. Assim, buscamos definir a ideia de homotopia intervalar, tanto considerando a Scott-continuidade, quanto a Moore-continuidade. Formalmente falando, desejamos interpretar como a noção de deformação contínua de uma função $f$ em uma outra $g$, com mesmo domínio e contradomínio - realizado matematicamente através da construção de uma função contínua (homotopia) $H$ parametrizada no intervalo real $[0,1]$, que, para cada $t \in$ $[0,1]$, define uma função contínua com mesmo domínio e contradomínio de $f$ e $g$, coincidindo com $f$, para $t=0$, e com $g$, para $t=1$ - se comporta quando pensamos na continuidade como sendo a continuidade de Scott (ou Moore) (veja [13, 2]) para funções intervalares.

Como resultado, demonstramos algumas propriedades que a homotopia intervalar goza, além de relacioná-las com outros conceitos diretamente ligados à matemática intervalar, tais como, representação intervalar (representação canônica). Além disso, demonstramos que, a menos de homeomorfismos, a reta real $\mathbb{R}$ tem o mesmo tipo de homotopia que $\mathbb{R}$ (conjunto dos intervalos fechados com extremos reais).

Alguns resultados são apresentados aqui sem demonstração, entretanto, uma prova formal para cada um deles pode ser encontrada em [13].

\section{Homotopia}

No que segue estamos sempre considerando $I=[0,1]$. Lembremos também que uma função $f$, entre espaços topológicos $X$ e $Y$, é contínua se, e somente se, a imagem inversa de conjunto aberto é um conjunto aberto, isto é, se $V$ é aberto em $Y$, então devemos ter $f^{-1}(V)$ aberto em $X$.

Definição 2.1. Sejam $f, g: X \longrightarrow Y$ duas funções contínuas entre os espaços topológicos $X$ e $Y$. Dizemos que $f$ e $g$ são homotópicas, e denotamos por $f \backsim g$, se existir uma função contínua $F: X \times I \longrightarrow Y$ tal que $F(x, 0)=f(x)$ e $F(x, 1)=$ $g(x)$, para todo $x \in X$. A função $F$ é chamada de homotopia entre $f$ e $g$.

Vale observar que, uma homotopia pode ser pensada como sendo uma deformação contínua de $f$ em $g$, com parâmetro $t$, isto é, para cada $t \in I, F(x, t)$ é uma função contínua de $X$ em $Y$. Dessa forma, uma outra maneira de representar uma homotopia é fazendo $F(x, t)=F_{t}(x)$ para cada $t \in I$. Nesse caso, $F_{0}(x)=f(x)$ e $F_{1}(x)=g(x)$. Faremos uso das duas notações no decorrer do texto, de acordo com a necessidade ou conveniência em cada caso.

Exemplo 2.1. Sejam $\mathbb{R}$ o conjunto dos números reais e $f, g: X \longrightarrow \mathbb{R}^{2}$ duas funções contínuas. É fácil verificar que a aplicação dada por $F(x, t)=(1-t) f(x)+$ $\operatorname{tg}(x)$ é uma homotopia entre $f$ e $g$, a qual chamamos de homotopia linear. Note que, $F$ é, para cada $x \in X$, exatamente o segmento de reta que une os pontos $f(x)$ e $g(x)$ (veja figura abaixo).

Lema 2.1. A relação $\simeq$ é de equivalência.

Demonstração. Lembremos primeiramente que uma relação de equivalência é uma relação $R$ que satisfaz as seguintes propriedades: 


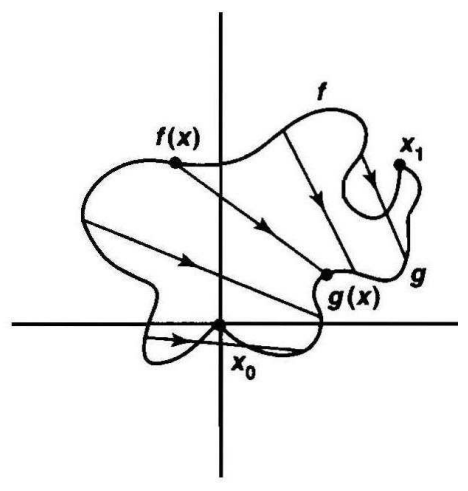

Figura 1: Homotopia linear entre $f$ e $g$ (Munkres [10])

- (Reflexividade) Para todo $x, x R x$;

- (Simetria) Se $x R y$, então $y R x$;

- (Transitividade) Se $x R y$ e $y R z$, então $x R z$.

Logo, a relação $\simeq$ é claramente reflexiva, isto é, para toda função contínua $f$, tem-se $f \simeq f$, posto que a aplicação $F$ dada por $F(x, t)=f(x)$, para todo $t \in I$, faz o papel dessa homotopia. Ainda, facilmente se verifica que, se $F$ é uma homotopia entre as funções $f$ e $g$, então $G(x, t)=F(x, 1-t)$ é uma homotopia entre $g$ e $f$. Resta portanto verificar a transitividade.

Assim, suponhamos que $f \simeq g$ e $g \simeq h$ e que $F$ e $G$ sejam, respectivamente, essas homotopias. Logo, a aplicação dada por

$$
H(x, t)=\left\{\begin{array}{rr}
F(x, 2 t), & \text { se } t \in[0,1 / 2] \\
G(x, 2 t-1), & \text { se } t \in[1 / 2,1]
\end{array}\right.
$$

é uma homotopia entre $f$ e $h$, como queríamos demonstrar.

\section{Scott e Moore Continuidade para Intervalos}

Consideremos o conjunto dos números reais $\mathbb{R}$. A partir dele podemos definir o conjunto $\mathbb{I}(\mathbb{R})=\{[a, b] \mid a, b \in \mathbb{R} e a \leqslant b\}$ de todos os intervalos em $\mathbb{R}$. Em 1962, Moore definiu sobre esse conjunto uma métrica $\operatorname{di}(X, Y)=\max (|\underline{x}-\underline{y}|,|\bar{x}-\bar{y}|)$, onde $X=[\underline{x}, \bar{x}]$ e $Y=[y, \bar{y}]$, a qual foi batizada com seu nome. Como é natural, a partir de uma noção de distância (métrica) é possível estabelecer a ideia de continuidade.

Definição 3.1. Uma função intervalar $f: \mathbb{I}(\mathbb{R}) \longrightarrow \mathbb{I}(\mathbb{R})$ é dita Moore-contínua se, para todo $X \in \mathbb{I}(\mathbb{R})$ e todo $\varepsilon>0$, existir um $\delta>0$ tal que, para todo $Y \in \mathbb{I}(\mathbb{R})$ se $\operatorname{di}(X, Y) \leqslant \delta$ então $\operatorname{di}(f(X), f(Y)) \leqslant \varepsilon$. 
Exemplo 3.1. A função dada por $M(X)=m(X)+\frac{(X-m(X))}{2}$, onde $m(X)=$ $\frac{a+b}{2}$ para todo $X=[a, b] \in \mathbb{I}(\mathbb{R})$, é Moore-contínua (veja [13]). Note que, a função $M$ é intervalar, considerando que todo intervalo pode ser escrito como a soma de um número real com um intervalo simétrico da seguinte forma: $X=[a, b]=m(X)+W$, onde $W=\frac{1}{2}(b-a)[-1,1]$.

Por outro lado, é possível definir uma outra noção de continuidade para funções intervalares, a Scott-continuidade. Para isso, basta considerarmos em $\mathbb{I}(\mathbb{R})$ a ordem de informação dada por $[a, b] \sqsubseteq[c, d]$ se, e somente se, $a \leqslant c \leqslant d \leqslant b$, para todo $[a, b],[c, d] \in \mathbb{I}(\mathbb{R})$.

Definição 3.2. Dizemos que uma função $f: \mathbb{I}(\mathbb{R}) \longrightarrow \mathbb{I}(\mathbb{R})$ é Scott-contínua se para todo conjunto dirigido ${ }^{4} \Delta$ de $\mathbb{I}(\mathbb{R}), f(\bigsqcup \Delta)=\bigsqcup f(\Delta)^{5}$.

Observação 3.1. Note que, toda função intervalar $F$ Scott-contínua é naturalmente inclusão monotônica, isto é, $F(X) \sqsubseteq F(Y)$ sempre que $X \sqsubseteq Y$. Vale ressaltar que desse ponto em diante, a expressão "inclusão"faz referência a inclusão de intervalos com relação à ordem de informação. Lembremos ainda que, uma função real $f$ é dita monotônica, se para todo $x$ e y tais que $x \leqslant y$, tem-se $f(x) \leqslant f(y)$.

Exemplo 3.2. (Santiago [13]) A função $F: \mathbb{I}(\mathbb{R}) \longrightarrow \mathbb{I}(\mathbb{R})$ definida por

$$
F(X)=\left\{\begin{aligned}
{[-1,1], } & \text { se } 0 \in X \\
{[0,0], } & \text { demais casos }
\end{aligned}\right.
$$

é Scott-contínua. Com efeito, $F$ é naturalmente monotônica. Além disso, suponhamos que $\Delta$ seja um conjunto dirigido. Se $0 \in \bigsqcup \Delta$ então $F(\bigsqcup \Delta)=[-1,1]$ e também, para cada $X \in \Delta$ vale $0 \in X$, de modo que $F(X)=[-1,1]$. Logo $\bigsqcup F(\Delta)=[-1,1]$. Por outro lado, se $0 \notin \bigsqcup \Delta$, então $F(\bigsqcup \Delta)=[0,0]$ e, para algum $X \in \Delta$ com $0 \notin X$, tem-se $F(X)=[0,0]$. Portanto, $F(\Delta)=\{[-1,1],[0,0]\}$ ou $F(\Delta)=\{[0,0]\}$. Em ambos os casos, $\bigsqcup F(\Delta)=[0,0]$.

\section{Representação}

Definição 4.1. Uma função intervalar $F$ é correta com relação a uma função real $f$ se satisfaz a seguinte propriedade: $x \in[a, b] \Rightarrow f(x) \in F([a, b])$. Neste caso, dizemos também que $F$ é uma representação intervalar de $f$.

Um fato importante, que devemos ressaltar, é que nem toda função real admite um representação intervalar. A exemplo disso, para a função

$$
f(x)= \begin{cases}\frac{1}{x}, & \text { se } x>0 \\ 1, & \text { demais casos }\end{cases}
$$

\footnotetext{
${ }^{4}$ Dado um poset $\langle P, \leqslant\rangle$, um subconjunto $\Delta \neq \emptyset$ de $P$ é dito um conjunto dirigido, se cada par de elementos de $\Delta$ possui um majorante.

${ }^{5} \sqcup \Delta$ é o supremo do conjunto dirigido $\Delta$. No caso de $\langle\mathbb{I}(\mathbb{R}), \sqsubseteq\rangle$, existe sempre $\sqcup \Delta$.
} 
não é possível definir um representação intervalar $F$ tal que, para todo $x \in[-1,1]$, $f(x) \in F([-1,1])$, visto que $f$ possui assíntotas. Por esse motivo, nos restringiremos daqui por diante à classe de funções não assintóticas.

Lema 4.1. Toda função monotônica (com relação à inclusão) $F: \mathbb{I}(\mathbb{R}) \longrightarrow \mathbb{I}(\mathbb{R})$ representa alguma função real $f$.

Corolário 4.1. Toda função intervalar F Scott-contínua representa alguma função real $f$.

Definição 4.2. Dada $f: \mathbb{R} \longrightarrow \mathbb{R}$, se $f$ é uma função real não-assintótica, a função $\widehat{f}([a, b])=[\min f([a, b]), \max f([a, b])]$ para todo $[a, b] \in \mathbb{I}(\mathbb{R})$, é chamada representação canônica de $f$.

De um ponto de vista mais informal, a representação canônica de uma função real $f$ vale exatamente o menor intervalo contendo $f([a, b])$, para cada intervalo $[a, b] \in \mathbb{I}(\mathbb{R})$.

Lema 4.2. Se $f: \mathbb{R} \longrightarrow \mathbb{R}$ é contínua, então para todo $[a, b] \in \mathbb{R}$, tem-se que $\widehat{f}([a, b])=f([a, b])$.

Corolário 4.2. Se $f: \mathbb{R} \longrightarrow \mathbb{R}$ é contínua e $y \in \widehat{f}([a, b])$, então $f^{-1}(y) \cap[a, b] \neq \emptyset$.

O colorário acima aponta para uma consequência importante do fato de termos $\widehat{f}([a, b])=f([a, b])$, quanto $f$ é contínua, o de que $f$, quando restrita ao intervalo $[a, b]$, é sobrejetiva.

Proposição 4.1. Para toda representação intervalar $F$ de uma função real $f$, temse que $F \sqsubseteq \widehat{f}^{6}$.

Observação 4.1. Em geral, Scott-continuidade e Moore-continuidade não são conceitos equivalentes. A exemplo disso, em [13] Santiago et al. mostram que a função intervalar $M$ apresentada no Exemplo 3.1 é Moore-contínua, mas não Scottcontinua, enquanto que a $F$ do Exemplo 3.2 refere-se a contrapositiva desse fato. Entretanto, se as funções intervalares em questão forem representações canônicas de funções contínuas, então esses conceitos coincidem. O teorema a seguir estabelece essa relação.

Teorema 4.1. (Teorema da Representação) Seja $f$ uma função real. Então,

1. f é contínua se e somente se $\widehat{f}$ é Scott-contínua.

2. $f$ é contínua se e somente se, $\widehat{f}$ é Moore-contínua.

\footnotetext{
${ }^{6}$ Para quaisquer funções intervalares $F$ e $G$ definidas em $\mathbb{R}$, dizemos que $F \sqsubseteq G$ se, para todo $X \in \mathbb{R}$, vale que $F(X) \sqsubseteq G(X)$.
} 


\section{Homotopia Intervalar}

Desenvolvemos nessa seção, o principal objetivo desse trabalho, isto é, apresentamos aqui a noção de homotopia intervalar, bem como a prova de alguns fatos relacionados a esse conceito.

A propriedade forte por trás da definição de homotopia, como podemos perceber na Definição 2.1, é a continuidade. Logo, é perfeitamente plausível pensarmos em definir homotopia dentro do ambiente das funções intervalares. Entretanto, precisamos tomar o cuidado especial de especificar o "tipo"de continuidade que estamos considerando, tendo em vista que no ambiente intervalar, desfrutamos de pelo menos dois conceitos de continuidade, a Scoot e a Moore, como vimos anteriormente. Isso provoca uma bifurcação na maneira de se definir homotopia, como veremos a seguir.

Definição 5.1. Sejam $f, g: \mathbb{I}(\mathbb{R}) \longrightarrow \mathbb{I}(\mathbb{R})$ duas funções Scott-contínuas. Uma aplicação $G: \mathbb{I}(\mathbb{R}) \times I \longrightarrow \mathbb{I}(\mathbb{R})$ é dita uma homotopia Scott-intervalar (SI-homotopia) entre $f$ e $g$ se, $G$ é Scott-continua, $G_{0}([a, b])=f([a, b])$ e $G_{1}([a, b])=g([a, b])$ para todo $[a, b] \in \mathbb{I}(\mathbb{R})$. Neste caso, dizemos que $f$ e $g$ são SI-homotópicas e denotamos por $f \simeq_{S I} g$.

Exemplo 5.1. Definamos a função

$$
G(X)=\left\{\begin{aligned}
{[-2,2], } & \text { se } 0 \in X \\
{[0,0], } & \text { demais casos }
\end{aligned}\right.
$$

Então, a aplicação $H: \mathbb{I}(\mathbb{R}) \times I \longrightarrow \mathbb{I}(\mathbb{R})$ tal que, a cada $t \in I$, satisfaz: $H_{t}(X)=$ $[-1-t, 1+t]$ se $0 \in X$ e $H_{t}(X)=[0,0]$ caso contrário, para todo $X \in \mathbb{I}(\mathbb{R})$, é uma SI-homotopia entre a função $F$ do Exemplo 3.2 e a função $G$.

Definição 5.2. Sejam $f, g: \mathbb{I}(\mathbb{R}) \longrightarrow \mathbb{I}(\mathbb{R})$ duas funções Moore-contínuas. Uma aplicação $G: \mathbb{I}(\mathbb{R}) \times I \longrightarrow \mathbb{I}(\mathbb{R})$ é dita uma homotopia Moore-intervalar entre $f$ e g, MI-homotopia por simplicidade, se $G$ é Moore-contínua, $G_{0}([a, b])=f([a, b])$ e ainda $G_{1}([a, b])=g([a, b])$ para todo $[a, b] \in \mathbb{I}(\mathbb{R})$. Neste caso, dizemos que $f$ e $g$ são MI-homotópicas e denotamos por $f \simeq_{M I} \mathrm{~g}$.

Exemplo 5.2. Dadas as funções $M$ do Exemplo 3.1 e a identidade $I d_{\mathbb{I}(\mathbb{R})}$ sobre $\mathbb{I}(\mathbb{R})$, a aplicação $L: \mathbb{I}(\mathbb{R}) \times I \longrightarrow \mathbb{I}(\mathbb{R})$, tal que, para cada $t \in I$, é dada por $L_{t}(X)=(1-t) M(X)+t I d_{\mathbb{I}(\mathbb{R})}(X)$ para todo $X \in \mathbb{I}(\mathbb{R})$, define uma MI-homotopia entre elas.

Observação 5.1. As relações $\simeq_{M I} e \simeq_{S I}$, por sua vez, também são relações de equivalência, e uma demonstração, semelhante à feita no Lema 2.1, pode ser dada para esses casos.

Lema 5.1. Se $H$ é uma homotopia no sentido usual, então $\widehat{H}_{t}=\widehat{H}_{t}$ para $t \in I$.

Demonstração. Para todo $[a, b] \in \mathbb{I}(\mathbb{R})$ e $t \in I$, temos

$$
\begin{aligned}
\widehat{H}_{t}[a, b] & =\widehat{H}([a, b], t) \\
& =[\min H([a, b], t), \max H([a, b], t)] \\
& =\left[\min H_{t}[a, b], \max H_{t}[a, b]\right] \\
& =\widehat{H}_{t}[a, b]
\end{aligned}
$$


Tendo em vista que as definições de Scott-continuidade e Moore-continuidade não são equivalente, é de se esperar que as noções de SI-homotopia e MI-homotopia também não coincidam. De fato, da Observação 4.1, podemos concluir que a aplicação $G$ do Exemplo 5.1 é uma SI-homotopia, mas não é uma MI-homotopia, enquanto que a homotopia $L$ do Exemplo 5.2 faz o papel inverso.

Entretanto, mais uma vez, no mundo das representações canônicas, esses conceitos coincidem, como podemos ver no teorema abaixo.

Teorema 5.1. Sejam $f, g: \mathbb{R} \longrightarrow \mathbb{R}$ duas funções contínuas e $\widehat{f}, \widehat{g}$ suas representações canônicas, respectivamente. Se $H$ é uma homotopia entre $f$ e g, então a representação canônica de $H$, dada por $\widehat{H}_{t}[a, b]=\left[\min H_{t}[a, b], \max H_{t}[a, b]\right]$ é uma homotopia Scott-intervalar (Moore-intervalar) entre $\widehat{f}$ e $\widehat{g}$.

Demonstração. Como, para cada $t \in I, H_{t}$ é contínua, então, do Teorema 4.1, segue que $\widehat{H_{t}}$ é Scott-contínua (Moore-contínua). Além disso, como, do Lema 5.1, sabemos que $\widehat{H}_{t}=\widehat{H}_{t}$, então $\widehat{H}$ é também Scott-contínua (Moore-contínua). Além disso, temos que

1. $\widehat{H}_{0}[a, b]=\left[\min H_{0}[a, b], \max H_{0}[a, b]\right]=[\min f[a, b], \max f[a, b]]=\widehat{f}[a, b]$

2. $\widehat{H}_{1}[a, b]=\left[\min H_{1}[a, b], \max H_{1}[a, b]\right]=[\min g[a, b], \max g[a, b]]=\widehat{g}[a, b]$

Portanto, de 1. e 2. e da Definição 5.1 (Definição 5.2), segue que $\widehat{H}$ é uma homotopia Scott-intervalar (Moore-intervalar).

Devido ao que foi estabelecido no teorema acima, no que segue, quando estivermos falando de homotopia entre representações canônicas, usaremos simplesmente o termo homotopia intervalar, a menos dos casos em se fizerem necessárias as devidas distinções.

Observação 5.2. Seja $H$ uma homotopia entre as funções reais $f$ e $g$. Um importante consequência da Proposição 4.1, é que $F \sqsubseteq \widehat{H}$, qualquer que seja a representação intervalar $F$ de $H$.

Agora, consideremos $H$ uma SI-homotopia entra duas funções intervalares $F$ e $G$ Scott-contínuas. Queremos provar que é possível construir uma homotopia entre convenientes funções reais $f$ e $g$ cuja representações intervalares são $F$ e $G$, respectivamente. Para isso, provemos as seguintes afirmações:

Afirmação I: $F$ e $G$ representam as funções reais $\underline{F}(x)=\min F([x, x])$ e $\underline{G}(x)=\min G([x, x])$ respectivamente, para todo $x \in \mathbb{R}$.

Com efeito, se $x \in X=[\underline{x}, \bar{x}]$ então $[x, x] \subseteq X$. Assim, temos que $F([x, x]) \subseteq$ $F(X)$ pois $F$ é monotónica, e consequentemente $\underline{F}(x)=\min F([x, x]) \in F(X)$, isto é, segue da Definição 4.1 que $F$ é uma representação intervalar de $\underline{F}$.

Afirmação II: As funções reais $\underline{F}$ e $\underline{G}$ são contínuas. 
Para provar esse fato, basta considerarmos em $\mathbb{I}(\mathbb{R})$ a quasi-métrica dada por $q i([\underline{x}, \bar{x}],[\underline{y}, \bar{y}])=\max \{\underline{y}-\underline{x}, \bar{x}-\bar{y}, 0\}$ definida por Acióly e Bedregal em [1], a qual permite formalizar a ideia de qi-continuidade ${ }^{7}$ que é equivalente à noção de Scott-continuidade para funções intervalares, como podemos ver em [12, 13].

Logo, sendo $x, y \in \mathbb{R}$, dado $\varepsilon>0$, existe $\delta>0$ tal que $q i([x, x],[y, y]) \leqslant \delta \Rightarrow$ $q i(F([x, x]), F([y, y])) \leqslant \varepsilon$. Por um lado,

$$
q i([x, x],[y, y]) \leqslant \delta \Rightarrow|x-y|<\delta
$$

Por outro lado,

$$
\begin{gathered}
q i(F([x, x]), F([y, y])) \leqslant \varepsilon \Rightarrow \max \{\underline{\mid F([y, y])}-\underline{F([x, x])}|,| \overline{F([x, x])}-\overline{F([y, y])} \mid, 0\}<\varepsilon \\
\Rightarrow \max \{|\underline{F}(y)-\underline{F}(x)|,|\bar{F}(x)-\bar{F}(y)|, 0\}<\varepsilon \Rightarrow|\underline{F}(x)-\underline{F}(y)|<\varepsilon, \text { isto é, temos } \\
q i(F([x, x]), F([y, y])) \leqslant \varepsilon \Rightarrow|\underline{F}(x)-\underline{F}(y)|<\varepsilon
\end{gathered}
$$

Portanto, segue das equações (5.1) e (5.2) que, para todo $\varepsilon>0$ dado, existe $\delta>0$ tal que, se $|x-y|<\delta$ tem-se $|\underline{F}(x)-\underline{F}(y)|<\varepsilon$, o que prova a continuidade de $\underline{F}$. Analogamente, verifica-se a continuidade de $\underline{G}$.

Assim, dada a SI-homotopia intervalar $H$ entre as funções intervalares $F$ e $G$, se tomarmos, para cada $t \in I, M_{t}(x)=H_{t}(x)=\min H_{t}([x, x])$ para todo $x \in \mathbb{R}$, a função $M: \mathbb{R} \times I \longrightarrow \mathbb{R}$ tal que $M(x, t)=\overline{=H}_{t}(x)$, é uma homotopia entre as funções reais $\underline{F}$ e $\underline{G}$. Logo, podemos enunciar a seguinte proposição.

Proposição 5.1. Seja $H$ uma SI-homotopia entre as funções intervalares Scottcontínuas $F$ e $G$. A função $M: \mathbb{R} \times I \longrightarrow \mathbb{R}$ tal que, para cada $t \in I, M_{t}(x)=$ $\min H_{t}([x, x]), x \in \mathbb{R}$, é uma homotopia entre $\underline{F}$ e $\underline{G}$.

Note que, a proposição acima não vale para funções intervalares Moore-contínuas, visto que não é possível garantir que uma função Moore-contínua qualquer seja a representação intervalar de alguma função real contínua. Para que o resultado acima fosse válido no ambiente das funções intervalares Moore-contínuas, teriamos que exigir que tanto $H$ quanto $F$ e $G$ fossem inclusões monotônicas.

Teorema 5.2. Sejam $f \simeq g$ e $h \simeq k$. Ent $\tilde{a} o \widehat{f} \circ \widehat{h} \simeq \widehat{g} \circ \widehat{k}$, desde que essas composições sejam possiveis.

Demonstração. Sejam $G$ a homotopia entre $f$ e $g$ e $K$ a homotopia entre $h$ e $k$. Assim a aplicação dada por

$$
H_{t}([a, b])=\left\{\begin{array}{rr}
h\left(G_{2 t}([a, b])\right), & \text { se } t \in[0,1 / 2] \\
K_{2 t-1}(g([a, b])), & \text { se } t \in[1 / 2,1]
\end{array}\right.
$$

é uma homotopia intervalar entre $\widehat{f} \circ \widehat{h}$ e $\widehat{g} \circ \widehat{k}$.

\footnotetext{
${ }^{7}$ Uma função $F: \mathbb{I}(\mathbb{R}) \longrightarrow \mathbb{I}(\mathbb{R})$ é $q i$-contínua, se $\forall X \in \mathbb{I}(\mathbb{R}), \forall \varepsilon>0, \exists \delta>0, \forall Y \in \mathbb{I}(\mathbb{R})$, $q i(X, Y) \leqslant \delta \Rightarrow q i(F(X), F(Y)) \leqslant \varepsilon$.
} 
Proposição 5.2. Sejam $f, g$ e $h$ funções reais contínuas. Se $H$ é uma homotopia entre $f$ e $g$ e $G$ é uma homotopia entre $g$ e $h$, então as representações intervalares de $H$ e $G$, dadas, respectivamente, por $\widehat{H}_{t}([a, b])=\widehat{H}_{t}([a, b])$ e $\widehat{G}_{t}([a, b])=\widehat{G}_{t}([a, b])$, para cada $t \in I$, são tais que $\widehat{G \circ H}_{t}=\widehat{G}_{t} \circ \widehat{H}_{t}$.

Demonstração. Levando em consideração que $(G \circ H)_{t}([a, b])=G_{t}\left(H_{t}([a, b])\right)$, para todo $[a, b] \in \mathbb{I}(\mathbb{R})$, então,

$$
\begin{aligned}
& \widehat{G}_{t} \circ \widehat{H}_{t}([a, b])=\widehat{G}_{t}\left(\widehat{H}_{t}([a, b])\right) \\
& =\widehat{G}_{t}\left(\widehat{H_{t}}([a, b])\right) \quad \text { pelo Lema } 5.1 \\
& =\widehat{G}_{t}\left(H_{t}([a, b])\right) \quad \text { pelo Lema } 4.2 \\
& =\widehat{G_{t}}\left(H_{t}([a, b])\right) \quad \text { pelo Lema } 5.1 \\
& =G_{t}\left(H_{t}([a, b])\right) \quad \text { pelo Lema } 4.2 \\
& =(G \circ H)_{t}([a, b]) \\
& =(\widehat{G \circ H})_{t}([a, b]) \quad \text { pelo Lema } 4.2 \\
& =\widehat{G \circ H}_{t}([a, b])
\end{aligned}
$$

Em geral, dadas duas funções reais $f$ e $g$ componíveis, a identidade $\widehat{f \circ g}=\widehat{f} \circ \widehat{g}$ não vale, isto é, a representação canônica não preserva composição. A exemplo disso, consideremos as funções reais $f(x, y)=x \cdot y$ e $g(x)=(x, x)$ para todo $x, y \in \mathbb{R}$. Logo, suas representantes canônicas são $\widehat{f}(X, Y)=X \cdot Y=[\min W, \max W]$ onde $W=\{\underline{x} \cdot \underline{y}, \underline{x} \cdot \bar{y}, \bar{x} \cdot \underline{y}, \bar{x} \cdot \bar{y}\}$ e $\widehat{g}(X)=([\min g(X), \max g(X)],[\min g(X), \max g(X)])$ para todo $X=[\underline{x}, \overline{\bar{x}}], Y=[\underline{y}, \bar{y}] \in \mathbb{I}(\mathbb{R})$, respectivamente. Portanto, por um lado, temos

$$
\widehat{f} \circ \widehat{g}(X)=\widehat{f}([\min g(X), \max g(X)],[\min g(X), \max g(X)])=[\min M, \max M]
$$

onde $M$ é como em $W$. Por outro lado,

$$
\widehat{f \circ g}(X)=\left[\min X^{2}, \max X^{2}\right]
$$

o que nos permite concluir que $\widehat{f \circ g} \neq \widehat{f} \circ \widehat{g}$.

A proposição acima mostra que esse tipo de problema não acontece em nível de homotopia intervalar.

\section{Retrato por Deformação}

Seja $M$ um subespaço do espaço topológico $X$. Dizemos que $M$ é um retrato por deformação de $X$ se a aplicação identidade sobre $X$ é homotópica a uma aplicação que leva todos os elementos de $X$ para $M$, de forma que, todos os pontos de $M$ permaneçam fixos durante a homotopia. Isso significa dizer que, existe uma homotopia $H: X \times I \longrightarrow X$ tal que $H(x, 0)=x$ e $H(x, 1) \in M$ para todo $x \in X$. A aplicação $r: X \longrightarrow M$ definida por $r(x)=H(x, 1)$ é uma retração de $X$ em $M$ e $H$ é uma homotopia entre a aplicação identidade em $X$ e $j \circ r$, onde $j: M \longrightarrow X$ é a inclusão. 
Claramente, o conceito de retrato por deformação é aplicável no ambiente intervalar. Se trocarmos a homotopia pela homotopia intervalar (tanto no sentido de Scott quanto no de Moore), podemos dizer que $M$ é um retrato por deformação de $\mathbb{I}(\mathbb{R})$ se existir uma homotopia intervalar $H$ entre $I d_{\mathbb{I}(\mathbb{R})}$ e a função $j_{I} \circ r_{I}: \mathbb{I}(\mathbb{R}) \longrightarrow \mathbb{I}(\mathbb{R})$, onde $r_{I}$ e $j_{I}$ são versões intervalares de uma dada retração $r$ e de uma inclusão $j$, respectivamente.

Consideremos o subconjunto $\operatorname{Tot}(\mathbb{I}(\mathbb{R}))=\{[x, x] \mid x \in \mathbb{R}\}$ de $\mathbb{I}(\mathbb{R})$. Não é difícil verificar que $\operatorname{Tot}(\mathbb{I}(\mathbb{R}))$ quando restrito à ordem de $\mathbb{I}(\mathbb{R})$, possui o mesmo tipo de estrutura que $\mathbb{I}(\mathbb{R})$.

Teorema 6.1. O conjunto Tot $(\mathbb{I}(\mathbb{R}))$ é um retrato por deformação de $\mathbb{I}(\mathbb{R})$.

Demonstração. Definamos a aplicação $H: \mathbb{I}(\mathbb{R}) \times I \longrightarrow \mathbb{I}(\mathbb{R})$ por

$$
H_{t}([a, b])=\left\{\begin{aligned}
{[(1-t) a, b], } & \text { se } t \neq 1 \\
{[b, b], } & \text { se } t=1
\end{aligned}\right.
$$

Precisamos mostrar que $\bigsqcup H_{t}(\Delta)=H_{t}(\bigsqcup \Delta)$ para todo subconjunto dirigido $\Delta$ de $\mathbb{I}(\mathbb{R})$. Recordemos que

- $[a, b] \sqsubseteq[c, d]$ se e somente se $a \leqslant c \leqslant d \leqslant b$.

- Denotando $X=[\underline{x}, \bar{x}]$, temos que $\bigsqcup \Delta=[\underline{\bigsqcup \Delta, \bar{\square}}]$ onde $\underline{\bigsqcup \Delta}=\sup \{\underline{x} \mid X \in$ $\Delta\}$ e $\bar{\square}=\inf \{\bar{x} \mid X \in \Delta\}$.

Assim, suponhamos que $\Delta$ seja um conjunto dirigido e seja $X=[\underline{x}, \bar{x}] \in \Delta$ arbitrário. Temos que, para $t \neq 1, H_{t}(\bigsqcup \Delta)=H_{t}([\bigsqcup \Delta, \overline{\square \Delta}])=[(1-t) \bigsqcup \Delta, \overline{\square \Delta}]$ e $H_{t}(X)=[(1-t) \underline{x}, \bar{x}]$ para todo $X \in \Delta$.

Como $\bigsqcup \Delta \sqsupseteq X$, qualquer que seja $X \in \Delta$, então $[\bigsqcup \Delta, \bar{\square}] \sqsupseteq[\underline{x}, \bar{x}]$ e portanto, $\underline{x} \leqslant \bigsqcup \Delta \leqslant \overline{\bigsqcup \Delta} \leqslant \bar{x}$. Logo, $(1-t) \underline{x} \leqslant(1-t) \bigsqcup \Delta \leqslant \overline{\bar{\square}} \leqslant \bar{x}$ visto que $1-t>0$, o que nos permite concluir que

$$
[(1-t) \bigsqcup \Delta, \overline{\bigsqcup \Delta}] \sqsupseteq[(1-t) \underline{x}, \bar{x}]
$$

isto é, $\bigsqcup H_{t}(\Delta)$ é cota superior do conjunto $H_{t}(\bigsqcup \Delta)$.

Resta provar que $\bigsqcup H_{t}(\Delta)$ é a menor das cotas superiores de $H_{t}(\bigsqcup \Delta)$. Para isso, suponhamos que $[c, d]$ é tal que $[\underline{x}, \bar{x}] \sqsubseteq[c, d]$ para todo $X \in \Delta$. Então, por definição, sabemos que $\underline{x} \leqslant c \leqslant d \leqslant \bar{x}$. Como $\underline{\bigsqcup}=\sup \{\underline{x} \mid X \in \Delta\}$, segue que

$$
\bigsqcup \Delta \leqslant c
$$

Analogamente, verifica-se que

$$
d \leqslant \overline{\bigsqcup \Delta}
$$

Portanto, de (6.1) e (6.2), temos que $[\bigsqcup \Delta, \overline{\square \Delta}] \sqsubseteq[c, d]$, como queriamos demonstrar. 
Observação 6.1. Vale ressaltar que a homotopia intervalar $H$ exibida na demonstração da proposição acima não é única. Na verdade, existem diversas formas de construir convenientemente essa $H$ como podemos, por exemplo, citar

$$
H_{t}([a, b])=\left\{\begin{aligned}
{[a,(1-t) b], } & \text { se } t \neq 1 \\
{[a, a], } & \text { se } t=1 .
\end{aligned}\right.
$$

Dizemos que dois conjuntos $X$ e $Y$ possuem o mesmo tipo de homotopia se dadas funções contínuas $f: X \longrightarrow Y$ e $g: Y \longrightarrow X$ tivermos que $g \circ f$ é homotópica à identidade em $X$ e $f \circ g$ é homotópica à identidade em $Y$. Neste caso, $f$ e $g$ são ditas homotopicamente equivalentes e $g$ é chamada de inversa homotópica de $f$.

Note que, se $A$ é um retrato por deformação de $X$, então $A$ e $X$ têm o mesmo tipo de homotopia, visto que a composição $r \circ j$, entre a inclusão $j: A \longrightarrow X$ e a retração $r: X \longrightarrow A$, é igual à identidade em $A$, enquanto que $j \circ r$ é homotópica, por definição, à identidade em $X$. Logo, podemos concluir, a partir do teorema 6.1 , que $\operatorname{Tot}(\mathbb{I}(\mathbb{R}))$ tem o mesmo tipo de homotopia que $\mathbb{I}(\mathbb{R})$.

O interessante é que, considerando o importante fato de que $\operatorname{Tot}(\mathbb{I}(\mathbb{R}))$ é homeomorfo ao conjunto dos números reais $\mathbb{R}$ (veja [12]), podemos afirmar que, a menos de homeomorfismos, $\mathbb{I}(\mathbb{R})$ e $\mathbb{R}$ têm o mesmo tipo de homotopia.

\title{
7. Considerações Finais
}

A ideia de relacionar o conceito de homotopia e matemática intervalar (veja, por exemplo, [11]), apesar de ainda ter sido pouco explorada, parece ser muito interessante. Na verdade, constitui um verdadeiro mundo novo para se pesquisar, em virtude da teoria de homotopia já ser bastante desenvolvida e de ainda, por existirem poucos trabalhos que tratam especificamente desse tema na matemática intervalar.

Para trabalhos futuros, nosso interesse agora é introduzir o conceito de homotopia para funções intervalares definidas sobre reticulados, visto que a partir de um dado reticulado podemos construir uma versão intervalar dele. Além disso, gostaríamos também de atrelar a ideia de homotopia às t-normas intervalares, perfazendo assim um amplo campo de estudo (veja [2]).

\begin{abstract}
We present a mathematical formalization of the concept of homotopy to interval functions defined on the set of intervals with real extreme $\mathbb{I}(\mathbb{R})$ considering both Scott-continuity and the Moore-continuity.

We define what is an interval Scott homotopy as well as what is an interval Moore homotopy . Then we show that these two concepts coincide for canonical representation. Finally, we present some results involving interval homotopy and related concepts such as composition of homotopy deformation and portrait.
\end{abstract}

\section{Referências}

[1] B.M. Acióly, B.R.C. Bedregal, A quasi-metric topology compatible with inclusion monotonicity on interval space, Reliable Computing, 3, No. 3 (1997), 305-313. 
[2] B.R.C. Bedregal, A. Takahashi, The best interval representation of T-norms and automorphisms, Fuzzy Sets and Systems, 157, No. 24 (2006), 3220-3230.

[3] J.C. Burkill, Functions of intervals, Proceedings of the London Mathematical Society, 375-446, (1924).

[4] J. Dugundji, "Topology", Allyn and Bacon Inc., Boston, 1966.

[5] P.S. Dwyer, "Computation with Approximate Numbers", p. 11-34, Wiley and Sons, New York, 1951.

[6] L. Jaulin, M. Kieffer, O. Didrit, E. Walter "Applied Interval Analysis", Springer-Verlag, London, 2001.

[7] R.E. Moore, "Automatic Error Analysis in Digital Computation", Technical Report LMSD-4842, Lockheed Missiles and Space Division, Sunnyvale, California, 1959.

[8] R.E. Moore, "Interval Analysis", Prentice-Hall, Englewood Cliffs, NJ, 1966.

[9] R.E. Moore, R.B. Kearfott, M.J. Cloud,"Interval Analysis", Society for Industrial and Applied Mathematics, PA, 2009.

[10] J.R. Munkres, "Topology, a First Course", Prentice Hall, Englewood Cliffs, NJ, 1975.

[11] J. Piper, Interval Skeletons, In "Proceedings 11th IAPR Internacional Conference on Pattern Recognition", vol. III, 1992.

[12] R.H.N. Santiago, B.C. Bedregal, B.M. Acióly, Comparing continuity of interval functions based on Moore and Scott topologies, Eletronic Journal on Mathematics of Computation (2005), disponível em http://www.cin.ufpe.br/ejmc.

[13] R.H.N. Santiago, B.C. Bedregal, B.M. Acióly, Formal aspects of correctness and optimality of interval computations, Formal Aspects of Computing, 18 (2006), 231-243.

[14] T. Sunaga, Theory of an interval algebra and its application to numerical analysis, RAAG Memoirs, 2 (1958), 547-564.

[15] W. Taylor, Simple equations on real intervals, Algebra Univers., 61 (2009), 213-226.

[16] G.W. Whitehead, "Elements of Homotopy theory", Springer-Verlag, NY, 1978.

[17] M. Warmus, "Calculus of Approximations", Bull. Acad. Polon. Sci. Cl. III, 4 (1956), 463-464.

[18] R.C. Young, The algebra of many-valued quantities, Math. Ann., (1931), 260290. 